\title{
Assessment of clinical activity and severity using serum ANCA and ASCA antibodies in patients with ulcerative colitis
}

Yanhua Pang ${ }^{1 \dagger}$, Huijie Ruan ${ }^{2 \dagger}$, Dongfang Wu ${ }^{3 \dagger}$, Yanfei Lang ${ }^{2}$, Ke Sun ${ }^{4}$ and Cuiping $\mathrm{Xu}^{2 *}$

\begin{abstract}
Background: Ulcerative colitis (UC) is a chronic, non-specific inflammatory bowel disease (IBD) with unknown etiology. The lack of specific clinical manifestations, standard diagnostic criteria, objective and accurate indicators to the severity of the disease and the efficacy of the treatment, often results in difficulties in diagnosis and timely treatment of UC. Therefore, there is a need to develop a clinically suitable serum biomarker assay with high specificity and sensitivity.
\end{abstract}

Objective and methods: To explore the significance of anti-neutrophil cytoplasmic antibodies (ANCA) and antisaccharomyces cerevisiae antibodies (ASCA) in the diagnosis, differential diagnosis and treatment assessment in patients with ulcerative colitis (UC). Serum levels of ANCA-lgG, ASCA-lgA and ASCA-lgG were measured by an enzyme-linked immunosorbent assay (ELISA) in 105 UC patients, 52 non-UC patients and 100 healthy controls.

Results: (1) Both the ANCA-IgG level and its positive rate in UC patients were significantly higher than those in non-UC controls and healthy controls $(p<0.01)$. However, the levels of ASCA-IgA, ASCA-lgG and the positive rates in UC patients had no statistical differences when compared with those in non-UC controls or healthy controls $(p>0.05)$.

(2) The sensitivity of ANCA ${ }^{+}$and $\mathrm{ANCA}^{+} / \mathrm{ASCA}^{-}$in detecting $U C$ patients was $61.90 \%$ and $55.24 \%$, respectively, whereas the specificity was $91.45 \%$ and $94.08 \%$, respectively. The sensitivity of $\mathrm{ASCA}^{+}$and $\mathrm{ASCA}^{+} / \mathrm{ANCA}^{-}$in non-UC disease controls was $5.33 \%$ and $3.85 \%$, respectively, and specificity was $83.9 \%$ and $88.78 \%$, respectively. (3) When UC patients were grouped into mild, moderate or severe subtypes, the ANCA-lgG levels were correlated with the severity of $U C$, and the differences of the ANCA-IgG levels were statistically different among the three subtypes $(p<0.05)$.

There was no correlation between the levels of ANCA-lgG and the disease locations of UC.

Conclusions: (1) Serum levels of ANCA may be useful in the diagnosis of UC. (2) Dynamic quantitation of ANCA-lgG levels may be helpful in determining the severity of $U C$ and therefore, may guide treatment of $U C$.

Keywords: Ulcerative colitis, Anti-Saccharomyces cerevisiae antibody, Anti-neutrophil cytoplasmic antibody, Dynamic quantitation

*Correspondence: cuipingxu@hotmail.com

†Yanhua Pang, Huijie Ruan and Dongfang Wu contributed equally to this work

${ }^{2}$ Department of Gastroenterology, The First Hospital of Shanxi Medical University, Taiyuan, Shanxi 300001, China

Full list of author information is available at the end of the article

\section{Background}

In theory, quantitative measurements of UC-related auto-antibodies would be helpful for the clinical diagnosis and treatment assessment of UC. Antineutrophil cytoplasmic antibodies (ANCA) are a group of auto-antibodies with cytoplasmic components of the neutrophils as the target antigens, which can release lysozymes through capillaries, damage blood vessels and

c) The Author(s) 2020. This article is licensed under a Creative Commons Attribution 4.0 International License, which permits use, sharing, adaptation, distribution and reproduction in any medium or format, as long as you give appropriate credit to the original author(s) and the source, provide a link to the Creative Commons licence, and indicate if changes were made. The images or other third party material in this article are included in the article's Creative Commons licence, unless indicated otherwise in a credit line to the material. If material is not included in the article's Creative Commons licence and your intended use is not permitted by statutory regulation or exceeds the permitted use, you will need to obtain permission directly from the copyright holder. To view a copy of this licence, visit http://creativeco mmons.org/licenses/by/4.0/. The Creative Commons Public Domain Dedication waiver (http://creativecommons.org/publicdomain/ zero/1.0/) applies to the data made available in this article, unless otherwise stated in a credit line to the data. 
intestine tissues, and also cause tissue damages through T cell-mediated cellular immune synergy $[1,2]$.

In recent years, studies have demonstrated that these ANCAs specifically mediate several diseases such as glomerulonephritis, systemic vasculitis, nodular granulomatosis and autoimmune hepatitis. An antibody named as atypical nuclear ANCA (atypical p-ANCA) has been related to UC [3, 4]. Another antibody highly associated with IBD is anti-Saccharomyces cerevisiae antibody (ASCA) which is directed against the yeast genus. ASCA mainly targets at peptidemimetic polysaccharide on the cell wall of the yeast. The mechanisms of these antibodies involved in IBD may be related to the increased intestinal permeability of the disease and the exposure to immune response cells of yeast antibodies [5]. By detecting serum levels of ANCAIgG, ASCA-IgA and ASCA-IgG, we have investigated the clinical significance of these antibodies in UC diagnosis, differential diagnosis, and possible correlations of antibody levels to the disease state and to the treatment efficacy.

\section{Patients and methods Patients}

Blood samples from 105 UC patients (52 males, 53 females, average age of $47.33 \pm 15.43$ years old) diagnosed by the outpatient and inpatient of the First Hospital of Shanxi Medical University from July 2015 to October 2016 were included in this study. The diagnosis of UC was based on clinical, endoscopic and histopathological findings in accordance with the IBD diagnostic criteria determined by the Chinese Medical Association meeting in Guangzhou [6]). Blood samples from 52 non-UC patients (28 males, 24 females, average age of $50.9 \pm 14.0$ years old), which were diagnosed as other intestinal disease (i.e. colitis, terminal ileitis, intestinal tuberculosis, intestinal polyps, white's disease and crohn's disease) according to the colonoscopyexamination, were selected as disease control. Meanwhile, 100 blood samples from healthy volunteers who came from our health examination center (54 males, 46 females, average age of $53.2 \pm 14.9$ years old) used in this study. There were no significant differences in both gender and average age among the three groups. According to Mayo Score System, the 105 UC serum samples were divided into three subgroups based on the disease severity: mild group (49 cases), moderate group (40 cases) and severe group (16 cases) [7]. The basic clinical data of the selected samples were shown in Table 1.

\section{Serologic measurements}

Five $\mathrm{mL}$ of blood samples were drawn from UC patients, disease controls and healthy controls and then centrifuged at $3000 \mathrm{r} / \mathrm{min}$ for $10 \mathrm{~min}$. The serum samples were collected and stored at $-80^{\circ} \mathrm{C}$ until used. Three serum antibodies (ANCA-IgG, ASCA-IgA and ASCAIgG) were detected using ANCA-IgG, ASCA-IgA and ASCA-IgG ELISA kits manufactured by Shanxi Ruihao Biotechnology company (Shanxi, China), and all the assays were conducted according to the kit instructions. Based on the kit instruction, the cut-off values of ANCAIgG, ASCA-IgA and ASCA-IgG were 5.03, 1.91 and $7.31 \mathrm{U} / \mathrm{mL}$, respectively. Antibody concentration levels greater than the cut-off value were regarded as positive.

\section{Statistical analysis}

SPSS 19.0 software package was used for statistical analysis. All the results were presented as the mean \pm standard error $(\bar{x} \pm S)$. The comparisons were conducted using variance analysis, and the comparison between each pair of the three groups was conducted using SNK test. The enumeration data were expressed by percentage, and the data comparison between two groups used Fisher Exact Probability test. The difference was regarded as statistically significant when $p<0.05$.

Table 1 Basic clinical information, serum levels and positive rates of ANCA and ASCA of the patients and controls

\begin{tabular}{|c|c|c|c|c|c|c|c|c|}
\hline Patients groups & $\mathrm{N}$ & Male & Female & Male:Female & Average of age (yrs) & $\begin{array}{l}\text { ANCA-IgG } \\
\mathrm{N}(\%)\end{array}$ & $\begin{array}{l}\text { ASCA-IgA } \\
N(\%)\end{array}$ & $\begin{array}{l}\text { ASCA-IgG } \\
\text { N (\%) }\end{array}$ \\
\hline Healthy controls & 100 & 54 & 46 & $1: 0.85$ & $53.19 \pm 14.85$ & $\begin{array}{l}3.15 \pm 2.24 \\
6(6)\end{array}$ & $\begin{array}{l}1.16 \pm 1.49 \\
7(7)\end{array}$ & $\begin{array}{l}3.41 \pm 5.41 \\
8(8)\end{array}$ \\
\hline Disease controls & 52 & 28 & 24 & 1:0.86 & $50.88 \pm 14.01$ & $\begin{array}{l}4.03 \pm 5.96 \\
7(13.5)\end{array}$ & $\begin{array}{l}0.95 \pm 1.94 \\
3(5.77)\end{array}$ & $\begin{array}{l}2.38 \pm 3.16 \\
1(1.92)\end{array}$ \\
\hline UC & 105 & 52 & 53 & $1: 1.02$ & $47.33 \pm 15.43$ & $\begin{array}{l}17.5 \pm 30.6^{a, b} \\
65(61.9)^{a, b}\end{array}$ & $\begin{array}{l}2.06 \pm 9.18 \\
11(10.5)\end{array}$ & $\begin{array}{l}4.05 \pm 6.42 \\
13(12.4)\end{array}$ \\
\hline
\end{tabular}

Age years with mean $\pm S D$, antibody levels are $\mathrm{U} / \mathrm{mL}$ (mean $\pm \mathrm{SD})$

a $P<0.05$ : Compared with healthy controls

b $P>0.05$ : Compared with disease controls 


\section{Results}

Serum levels of ANCA, ASCA and the positive rates among the three comparison groups

Our data suggested that: (1) The ANCA-IgG level and positive rate in UC group were significantly higher than those of disease control group and healthy control group,the differences were statistically significant $(p<0.01)$; There were no significant differences in ANCAIgG level and positive rate between disease control group and healthy control group $(p>0.05)$; (2) There were no significant differences in ASCA-IgA, ASCA-IgG levels and positive rate among UC group, disease control group and healthy control group. Results were shown in Table 1.

\section{Correlations between serum autoantibody levels} and the severity of UC as well as the disease locations

The level of serum ANCA-IgG in severe group was significantly higher than those in moderate group and mild group $(p<0.01)$. The serum level of ANCA-IgG was also significant higher in moderate group than that in mild group $(p<0.05)$. There were no significant differences of the serum ASCA-IgA and ASCA-IgG levels in patients among the three subgroups. Results were shown in Table 2.

According to Montreal Classification [8], all the 105 UC serum samples were divided into three subgroups based on the lesion location such as the whole colon type group (42 cases), the left colon type group (32 cases) and the rectal type group (31 cases). The levels of serum ANCA-IgG, ASCA-IgA and ASCA-IgG had no significant differences among the three groups $(p>0.05)$. Results were shown in Table 3.

\section{Comparative evaluation of a single ANCA test vs. combinational tests of ASCAs for UC diagnosis and differential diagnosis}

Both assays' specificity and sensitivity were used for the comparative evaluation. The sensitivity of serum $\mathrm{ANCA}^{+}$ and $\mathrm{ANCA}^{+} / \mathrm{ASCA}^{-}$in $\mathrm{UC}$ group was $61.90 \%$ and $55.24 \%$, respectively, whereas the specificity was $91.45 \%$

Table 2 Serum levels of ANCA and ASCA in UC patients with different disease status

\begin{tabular}{lcccc}
\hline Patients groups & N & ANCA-IgG & ASCA-IgA & ASCA-IgG \\
\hline UC mild & 49 & $4.65 \pm 2.81$ & $3.36 \pm 13.2$ & $3.18 \pm 5.02$ \\
UC moderate & 40 & $14.70 \pm 18.4^{c}$ & $0.83 \pm 2.00$ & $4.47 \pm 7.11$ \\
UC severe & 16 & $64.00 \pm 52.1^{\mathrm{a}, \mathrm{b}}$ & $1.15 \pm 3.04$ & $2.86 \pm 6.01$ \\
\hline
\end{tabular}

Antibody levels are $\mathrm{U} / \mathrm{mL}$ (mean $\pm \mathrm{SD})$

a Comparison between UC severe group and UC moderate group $(p<0.01)$

b Comparison between UC severe group and UC mild group $(p<0.01)$

c Comparison between UC moderate group and UC mild group $(p<0.05)$
Table 3 Serum levels of ANCA and ASCA in UC patients with different disease locations

\begin{tabular}{lllll}
\hline Disease locations & N & ANCA-IgG & ASCA-IgA & ASCA-IgG \\
\hline Whole colon type & 42 & $19.90 \pm 36.00$ & $1.45 \pm 6.68$ & $4.36 \pm 6.68$ \\
Left colon type & 32 & $20.76 \pm 34.57$ & $3.45 \pm 15.90$ & $2.90 \pm 5.99$ \\
Rectal type & 31 & $10.97 \pm 13.15$ & $1.46 \pm 3.05$ & $3.36 \pm 5.12$ \\
\hline
\end{tabular}

The levels of serum ANCA and ASCA in patients within the whole colon type group, left colon type group and rectal type group were compared, and no significant difference with each other were observed $(p>0.05)$. Antibody levels are $\mathrm{U} / \mathrm{mL}($ mean $\pm \mathrm{SD})$

and $94.08 \%$, respectively. The sensitivity of serum $\mathrm{ASCA}^{+}$ and $\mathrm{ASCA}^{+} / \mathrm{ANCA}^{-}$in non-UC disease control group was $5.33 \%$ and $3.85 \%$, respectively, and the specificity was $83.90 \%$ and $88.78 \%$, respectively. The positive predictive values of the above antibodies were $83.33 \%, 86.57 \%$, $8.33 \%$ and $8.00 \%$, respectively, and the negative predictive values were $77.65 \%, 75.26 \%, 77.83 \%$ and $78.48 \%$, respectively. These results were tabulated in Table 4 .

\section{Discussion}

Ulcerative colitis (UC) is a chronic inflammatory bowel disease with onset most frequently in young adulthood. Many patients with UC have a lifetime of debilitating physical symptoms (e.g., urgent diarrhea, rectal bleeding, abdominal pain). UC is diagnosed by the combination of clinical pattern, colonoscopy, CT, MR and histological findings (basal plasmacytosis, diffuse crypt atrophy and distortion, villous surface irregularity and mucus depletion) [6]. mild-moderate fewer than 4-6 bowel movements per day, mild-moderate rectal bleeding, absence of constitutional symptoms, and absence of features suggestive of high inflammatory activity based upon Truelove and Witt's criteria 3 and the Mayo Clinic score. Patients with mild-moderate disease activity generally are at low risk of requiring colectomy. However, certain disease features may predict an aggressive disease course. These include extensive disease, severe endoscopic activity, extra-intestinal manifestations, and elevated inflammatory markers [9]. ANCA-associated vasculitides (AAV)includes granulomatosis with polyangiitis (GPA), eosinophilic granulomatosis with polyangiitis (EGPA) and microscopic polyangiitis (MPA). Few cases of vasculitis were found associated with IBD [10].

Searching for laboratory bio-indicators related to UC diagnosis and providing a simple, objective and accurate serum marker for diagnosis, differential diagnosis and efficacy assessment are the ultimate goal in clinical gastroenterology. It has been well documented that ANCA is associated with UC. At present, the most widely used technologies for ANCA detection are indirect 
Table 4 Comparative analyses of single ANCA test vs. combinational tests for ANCA/ASCA in the diagnosis of UC

\begin{tabular}{|c|c|c|c|c|}
\hline Antibody/disease & Sensitivity (\%) & Specificity (\%) & $\begin{array}{l}\text { Positive predictive } \\
\text { value (\%) }\end{array}$ & $\begin{array}{l}\text { Negative } \\
\text { predictive } \\
\text { value (\%) }\end{array}$ \\
\hline $\mathrm{ANCA}^{+} / \mathrm{UC}$ & 61.90 & 91.45 & 83.33 & 77.65 \\
\hline $\mathrm{ANCA}^{+} / \mathrm{ASCA}^{-} / \mathrm{UC}$ & 55.24 & 94.08 & 86.57 & 75.26 \\
\hline $\mathrm{ASCA}^{+} /$disease control group & 5.77 & 83.90 & 8.33 & 77.83 \\
\hline $\mathrm{ASCA}^{+} / \mathrm{ANCA}^{-} /$disease control group & 3.85 & 88.78 & 8.00 & 78.45 \\
\hline
\end{tabular}

More than one positive test of ASCA-lgG and ASCA-IgA subtypes was regarded positive; two negative tests of the subtypes were regarded negative

immunofluorescence (IIF) assay and ELISA. Since it is quite common that antinuclear antibodies (ANA) can be present at the same time when ANCA is detected with the qualitative IIF assay. It has been documented that $\mathrm{ANCA}^{+}$may be missed through IIF test due to the interference of ANA, while this can be overcome when a specific ELISA method is used.

In our previous studies, we have demonstrated that quantitative ELISA assaying ANCA levels has a good consistency with IIF and yet has a sensitivity superior to IIF [11]. According to a large number of reports, ANCA is present in $40 \%-85 \%$ of UC patients, $5 \%-10 \%$ of CD patients, and $0-5 \%$ of healthy controls [12]. Bansi et al. have reported that ANCA is detected in $42.4 \%$ of UC patients but only in 5\% of CD with a specificity of $98 \%$ and a sensitivity of $42 \%$, respectively, for diagnosing UC [13]. Liu et al. have also reported that ANCA is detected in $48.6 \%$ of UC patients and in $25 \%$ of CD patients and the sensitivity of single test of ANCA for diagnosing UC is $48.6 \%$ with a specificity of $92.9 \%$ [14]. Qiu et al. have demonstrated that ANCA was detected in 68.3\% of UC patients and only in $1.7 \%$ of healthy controls with a sensitivity of $65.9 \%$ and a specificity of $92.7 \%$, respectively, in a single test of ANCA for the diagnosis of UC [15]. In this report, our results indicate that ANCA positivity is of a good clinical reference value for UC diagnosis and, the augment of ANCA levels may improve the diagnostic accuracy of UC.

At present, it is still controversial whether ANCA levels in UC patient is associated with the severity of the disease. He et al. have reported that ANCA levels are associated with the severity of the disease. Their results have shown that ANCA positive UC patients have a higher rate of intestinal mucosal vasculitis than ANCA negative UC patients. In addition, the UC patients with high ANCA levels also have a severe pathological inflammation at the intestinal mucosa [16]. However, Yang et al. have reported conflicting results [17]. In order to evaluate the correlation, UC patients have been divided into mild, moderate and severe groups based on the disease severity. Following the aggravation of the disease, levels of ANCA-IgG show upward tendency and the serum ANCA-IgG levels are indeed higher in patients in the severe group than those in the moderate and mild groups ( $\mathrm{p}<0.001)$. Similarly, the serum ANCA-IgG levels are also significantly higher in moderate patients than those in the mild group $(\mathrm{p}<0.05)$.

A study reported that the positive rate of ASCA in CD patients was $39 \%-72 \%$, higher than that of UC and disease control patients $(4 \%-14 \%$ and $4 \%-14 \%$ respectively). In this study, the positive rates of ASCA -IgG and ASCA -IgA in UC patients were $13 \%$ and $11 \%$, respectively, with no significant difference from this research data. Studies have shown that the presence of ASCA is related to small bowel involvement (especially ileum) [18], while the disease site of UC is mainly located in the colorectal, which may be one reason for the low positive rate of ASCA in UC patients. In a prospective comparative study of ASCA in IBD patients in China and whites, it was mentioned that there was a significant difference in the mutation frequency of NOD2 gene (NOD2 gene is considered to be a strongly correlated gene of $\mathrm{CD}$ ) in $\mathrm{CD}$ among different ethnic groups. Currently, the sensitivity of Chinese people to ASCA detection is lower than that of white people [19].

\section{Conclusions}

Serum $\mathrm{ANCA}^{+}$levels are helpful in the diagnosis of UC, while combined tests of serum ANCA-IgG, ASCA-IgG, and ASCA-IgA levels are useful in differential diagnosis of UC. Furthermore, in UC patients, serum ANCA levels is correlated with the severity of the disease and, therefore, a dynamic monitoring of serum ANCA levels during treatment may relieve patients from repeated use of invasive diagnostic methods. The dynamic testing of serum antibody levels as mentioned above may also help monitor disease progression, guide for more effective clinical medications and reduce the recurrence of UC.

\section{Abbreviations}

ANCA: Anti-neutrophil cytoplasmic antibodies; ASCA: Anti-Saccharomyces cerevisiae antibodies; UC: Ulcerative colitis; IBD: Inflammatory bowel disease; ANA: Antinuclear antibodies. 


\section{Acknowledgements}

We thank Dr. Wen Jie Zhang of Shihezi University School of Medicine for his critical review and intellectual input.

\section{Authors' contributions}

PY, RH and WD performed study, analyzed the data and drafted the first version of the manuscript; LY and SK collected data; XP designed and supervised study. All authors read and approved the final manuscript.

\section{Funding}

None.

\section{Availability of data and materials}

The datasets used and/or analysed during the current study are available from the corresponding author on reasonable request.

\section{Ethics approval and consent to participate}

All studies met the requirements for Ethics Committee of First Hospital of Shanxi Medical University.

\section{Consent for publication}

All authors provided consent for publication.

\section{Competing interests}

The authors declare that they have no competing interests.

\section{Author details}

1 Department of Gastroenterology, Beijing Chaoyang Hospital, Capital Medical University, Chaoyang District, Beijing 100020, China. ${ }^{2}$ Department of Gastroenterology, The First Hospital of Shanxi Medical University, Taiyuan, Shanxi 300001, China. ${ }^{3}$ Department of Gastroenterology, Hebei Yanda Hospital, Hebei Medical University, Sanhe, Hebei 065201, China. ${ }^{4}$ Department of Pathology, Shihezi University School of Medicine, Shihezi, Xinjiang 832002, China.

Received: 5 August 2019 Accepted: 7 May 2020

Published online: 20 May 2020

\section{References}

1. Zhang $L L, X u Z$, Huang GM, et al. Research of clinical application of serum antibody detection in inflammatory bowel disease. China Prac Med. 2016;26(11):7-9.

2. Vergara T, Cofre P, Cifuentes $S$, et al. Presence of antineutrophil cytoplasmic antibodies (ANCA) and anti-Saccharomyces cerevisiae antibodies (ASCA) among patients with ulcerative colitis. Rev Med Chil. 2006;134(8):960-4.

3. Zhang J, Han Y. Research development of IBD serum markers. Med J National Defense Forces North China. 2007;19(2):19.

4. Schoepfer AM, Beglinger C, Straumann A, et al. Fecal Calprotectin correlates more closely with the simple endoscopic score for crohn's disease (SES-CD) than CRP, blood leukocytes, and the CDAI. Inflamm Bowel Dis. 2013;19(2):332-41.

5. Standaert-Vitse A, Jouault T, Vandewalle P, et al. Candida albicans is an immunogen for anti-Saccharomyces cerevisiae antibody markers of Crohn's disease. Gastroenterology. 2006;130(6):1764-75.

6. Pinjin $\mathrm{H}$, Jiaming $\mathrm{Q}$, Kaichun $\mathrm{W}$, et al. Digestive disease branch of Chinese Medical Association, consensus on diagnosis and treatment of inflammatory bowel disease in China. Theor Pract Intern Med. 2013;8(1):408-13.

7. D'haens G, Sandborn WJ, Feagan BG, et al. A review of activity indices and efficacy end point for clinical trial of medical therapy in adults with ulcerative colitis. Gastroenterology. 2007;132(2):763-86.

8. Satsangi J, Silverberg MS, Vermeire S, et al. The Montreal classification of inflammatory bowel disease: controversies, consensus, and implications. Gut. 2006;55(6):749-53.

9. Dias CC, Rodrigues PP, Costa-Pereira AD, Magro F. Clinical predictors of colectomy in patients with ulcerative colitis: systematic review and metaanalysis of cohort studies. J Crohn's Colitis. 2015;9(2):156-63.

10. Sy A, Khalidi N, Dehghan N, et al. Vasculitis in patients with inflammatory bowel diseases: a study of 32 patients and systematic review of the literature. Semin Arthritis Rheum. 2016;45(4):475-82.

11. Song BB, Zhao Y, Zhang CY, et al. The comparision between two ANCA detection methods. Lab Med. 2011;26(7):457-60.

12. Buckland MS, Mylonaki M, Rampton D, et al. Serological markers in inflammatory bowel disease: diagnostic utility and phenotypic correlation. Clin Diagn Lab Immunol. 2005;12(11):1328.

13. Bansi DS, Chapman RW, Fleming KA. Prevalence and diagnostic role of antineutrophil cytoplasmic antibodies in inflammatory bowel disease. Eur J Gastroenterol Hepatol. 1996;8(9):881-5.

14. Liu J, Cheng W, Wang H, et al. The diagnostic value of serum antibody detection in inflammatory bowel disease. Chin J Internal Med. 2008;47(8):668-9.

15. Qiu L, The significance of detection of perinuclear antineutrophil cytoplasmic antibodies in patients with ulcerative colitis. Zhejiang Clinical Medicine.

16. Bin He, Zhenyu Zhang, et al. The significance of ANCA in diagnosis of UC in ulcerativecolitis diagnosis. Clin Focus. 2008;23(10):746.

17. Yang Dong, Wang Zhen. Clinical value of IBD serum associated antibody assay. J Da-Lian Med University. 2010;132(3):343-7.

18. Kansal S, Catto-Smith AG, Boniface K, et al. Variation of gut mucosal microbiome with anti-Saccharomyces cerevisiae antibody status in pediatric crohn disease. Pediatr Gastroenterol Nutr. 2019;69(6):696-703.

19. Lawrance IC, Murray K, Hall A, et al. A prospective comparative study of ASCA and PANCA in Chinese and Caucasian IBD patients. Am J Gastroenterol. 2004;99(11):2186-94.

\section{Publisher's Note}

Springer Nature remains neutral with regard to jurisdictional claims in published maps and institutional affiliations.
Ready to submit your research? Choose BMC and benefit from:

- fast, convenient online submission

- thorough peer review by experienced researchers in your field

- rapid publication on acceptance

- support for research data, including large and complex data types

- gold Open Access which fosters wider collaboration and increased citations

- maximum visibility for your research: over $100 \mathrm{M}$ website views per year

At BMC, research is always in progress.

Learn more biomedcentral.com/submissions 